\title{
Contact Surround in Opportunistic Networks
}

\author{
Nadjet Belblidia ${ }^{\star}$, Marcelo Dias de Amorim${ }^{\star}$, Jérémie Leguay ${ }^{\dagger}$, Vania Conan ${ }^{\dagger}$, Jon Crowcroft ${ }^{\ddagger}$, and Serge Fdida ${ }^{\star}$ \\ * UPMC Paris Universitas \\ Paris, France \\ $\dagger$ Thales Communications \\ Colombes, France \\ $\ddagger$ Cambridge University \\ Cambridge, UK \\ first.last@lip6.fr \\ first.last@fr.thalesgroup.com \\ first.last@cl.cam.ac.uk
}

\begin{abstract}
Is the temporal dimension alone sufficient to characterize contacts in opportunistic networks? Several studies analyze the temporal aspect of contacts with significant results concerning contact and inter-contact distributions. Nevertheless, only the temporal dimension does not give a complete overview of contact characterization. In this paper, we propose the surround indicator as a metric to exhibit the contact's surrounding environment in opportunistic networks. We evaluate the surround indicator on two existing datasets and show that contacts have too heterogeneous and too unstable surrounds to be considered only in terms of duration. Besides a large variability of the surrounding environment within the duration of a single contact, it is frequent to observe contacts of identical duration that exhibit differences in their surrounds of more than a hundred times.
\end{abstract}

\section{Context And Problem Definition}

Important advances in the area of opportunistic networks have been achieved these latest years, including the proposal of routing and forwarding algorithms [1], [2], [3], [4], [5], the investigation of social relationships [6], [7], [8], [9], and the conception of applications to enable content sharing among users on the move [10], [11], [12]. The common point in all these contexts is that they rely on a thorough understanding of the properties governing contact patterns among users. This is a crucial step, as contacts in opportunistic networks may be characterized by a limited capacity due to, among others, user mobility, signal range, obstacles, and surrounding interferences. Providing fine characterization of contact capacity is however a difficult task, as it inherently depends on the physical conditions of the network and on the traffic demand.

A contact has two dimensions: temporal and spatial. The temporal dimension concerns the contact and intercontact distributions and has been the subject of several studies [13], [14], [15], [16], [17]. The spatial dimension of contacts is related to the contact surrounding environment. Although the surrounding environment has a direct impact on the contact capacity, it has been surprisingly underexplored in the literature. The few works that investigate this issue restrain themselves to the forwarding path characterization [18].

In this paper, we provide elements to the intuition that contacts should not be considered only in terms of duration. Fig. 1 illustrates two completely different situations where contacts last for the same duration. If we consider only the temporal dimension, both contacts are then of course identical. Nevertheless, although the contacts have the same duration, the one in Fig. 1(a) is clearly much more prone to interference as it happens in a denser zone. We argue

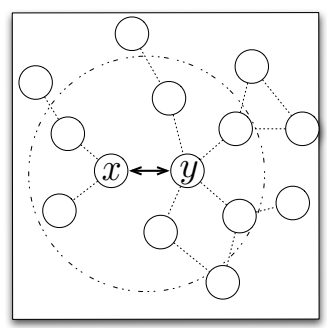

(a) Contact in a dense zone.

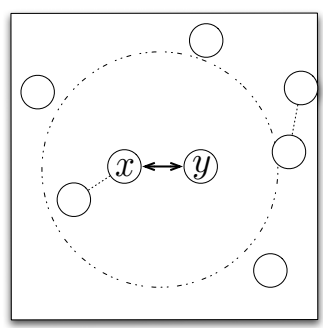

(b) Contact in a sparse zone.
Fig. 1. The same duration. Two situations.

that additional information providing simple indication on the contact surrounding conditions can bring a complementary knowledge for a better understanding of contact capacity.

We propose the surround indicator as a metric to exhibit the spatial dimension of contacts. The surround indicator gives explicit information about nearby contacts that are potential sources of interference with the contact under consideration. The surround indicator is computed from a directed graph representation of the network. Note that, although the proposed approach is merely indicative, such an estimation of the quality of a contact can be useful in a number of research problems. For example, to design more appropriate routing algorithms, to identify popular nodes, and to understand the network evolution through graph-related metrics, to cite a few. In this paper, we focus on both the characterization of opportunistic networks based on our metric and on the dynamics of the topology derived from this characterization.

We use the surround indicator to characterize the spatial dimension of contacts of two datasets collected in realworld experiments. First, we investigate the distribution of the surround indicator on a per-contact basis. Second, we examine the dynamics of the surrounding environment within the duration of a contact. Our idea here is to evaluate the common assumption that the topology remains static while two nodes meet. Our analyses reveal that:

- Opportunistic networking is much more dynamic than it is traditionally handled as. Besides the intermittent characteristic of connectivity, nodes also observe huge surround instability.

- Most of the time, we observe a high variability of the surround indicator within the same contact.

- In many cases, the surround indicator is expressed by 


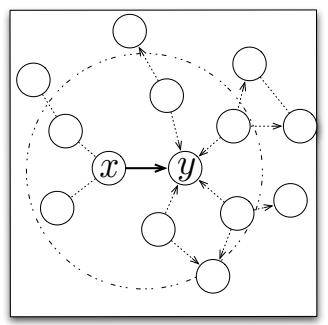

(a) Destination node in a dense zone.

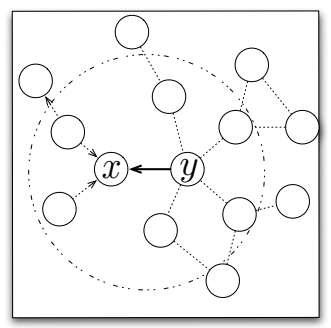

(b) Destination node in a sparse zone.
Fig. 2. Two directions. Different surrounds.

extreme values, indicating zones that are inherently prone to strong interference. This observation has fundamental practical impact, as for example in routing protocols based on the notion of node centrality (which might be in fact unfeasible in many situations).

These results highlight the importance of the spatial dimension of contacts in opportunistic networks.

The remainder of the paper is structured as follows. In Section II, we present our proposal for the surround indicator and discuss the practical utilization of this metric. We investigate then in Sections III and IV the surround and dynamics of contacts of two datasets collected in real-world experiments. Finally, we conclude the paper in Section $\mathrm{V}$ and give some directions for future work.

\section{EXHIBITING THE SPATIAL DIMENSION OF CONTACTS}

Principles. We refer to the situation represented in Fig. 1(a) to explain how the surround indicator is computed. We suppose that all contacts are bidirectional. In one hand, If node $x$ tries to send data to node $y$, the co-located contacts that may interfere with $y$ reception are the ingoing contacts of $y$ and also all their outgoing contacts, because of the omnidirectionality of wireless links. These contacts are represented with arrows in Fig 2(a). Similarly, if $y$ tries to send data to $x$, the contacts that may interfere with the $x$ reception are the ingoing contacts of $x$ and all their outgoing contacts (cf., Fig. 2(b)). We can see through this example that the surround indicator reflects the receiver surround. Moreover, the value corresponding to each direction of contact can be very different if the surrounding environment of the involved nodes are heterogeneous. In the following, we give a formal definition of the surround indicator.

Formal definition. Let $\mathbf{V}$ be the set of nodes in the network and $\overrightarrow{x y}$ be the directional contact between $x$ and $y$ during the interval $\left[t_{1}, t_{2}\right]$, with $x, y \in \mathbf{V}$. We refer to it as the target contact. $N_{x}^{+}(t)$ and $N_{x}^{-}(t)$ are respectively the sets of out-neighbors and in-neighbors of node $x$ at time $t$. Loosely speaking, we define the surround indicator $\mathcal{S}_{x y}(t)$ of the target contact $\overrightarrow{x y}$ at time $t \in\left[t_{1}, t_{2}\right]$ as the number of outgoing contacts of all $y$ 's in-neighbors except $x$ :

$$
\mathcal{S}_{x y}(t)=\sum_{\forall z \in N_{y}^{-}(t)-\{x\}}\left|N_{z}^{+}(t)\right|, \forall t \in\left[t_{1}, t_{2}\right] .
$$

Note that the surround indicator points out how many sources of interference (i.e., co-located contacts) might have an influence on the reception quality of the target contact. In the worse case, where the destination is the neighbor of all nodes and all the destination neighbors have outgoing link to all nodes (in a complete graph for example), $\mathcal{S}_{x y}(t)=$ $(N-1)(N-2)$ with $N=|V|$.

Interest. The surround indicator can be useful in a number of situations. A straightforward case is routing. For example, a possible policy would be to ignore small contacts with large surround indicators when alternative paths exist. A second case concerns bundle size; it could be useful for example to tune the size of a bundle in function of the surround indicators. The bundle is expected to find throughout the network. As a contact with a large surround indicator is much more prone to competitive flows, it could be better to try to transfer small messages to fairly share the bandwidth between co-located contacts. A third application is the detection of link popularity (as a complement to the notion of node popularity) based on the link's surround.

Dynamics. The surround indicator is likely to vary during the contact interval $\left[t_{1}, t_{2}\right]$, as some neighbors appear while others leave during the contact. We propose to decompose the contact into as many necessary sub-contacts with a stable surround indicator. Each sub-contact represents a different temporal view of a surround. We define the expansion as the number of sub-intervals associated to the same original contact.

This representation allows not only characterizing neighborhood density through the surround indicator, but also to have a clue on the network dynamics. Indeed, a high value of the expansion suggests frequent changes of the surround indicator value and reflects contact surround instability.

The creation of sub-intervals is illustrated in Fig. 3. The duration of contact $\overrightarrow{x y}$ is divided into as many sub-intervals as the number of changes in the neighborhood. Thus, in the example of Fig. 3, the contact surround evolution generates the division of the duration $[0,15]$ into six sub-intervals as follows :

$$
[\mathrm{x}, \mathrm{y}, 0,15] \rightarrow\left\{\begin{array}{c}
{[\mathrm{x}, \mathrm{y}, 0,1] ;[0]} \\
{[\mathrm{x}, \mathrm{y}, 1,3] ;[1]} \\
{[\mathrm{x}, \mathrm{y}, 3,5] ;[2]} \\
{[\mathrm{x}, \mathrm{y}, 5,10] ;[3]} \\
{[\mathrm{x}, \mathrm{y}, 10,14] ;[2]} \\
{[\mathrm{x}, \mathrm{y}, 14,15] ;[1]}
\end{array}\right.
$$

Therefore, the expansion is equal to six in this example. By investigating the dynamics within a contact, we somehow indirectly estimate the evolution of the contact capacity. Again, it is important to make it clear that our proposal is not a computation of the capacity, but an indication of the conditions under which a contact operates. We show in the following that 


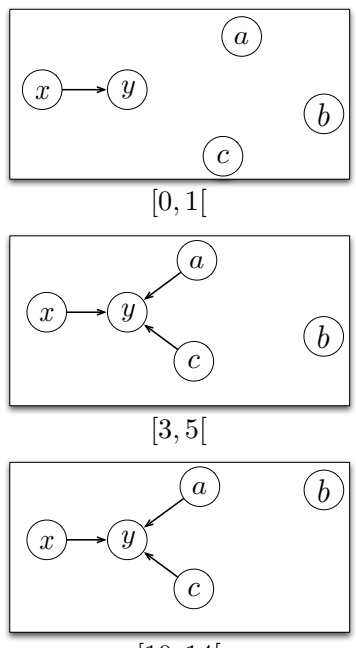

$[10,14[$

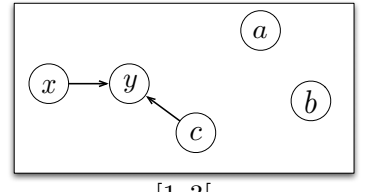

$[1,3[$

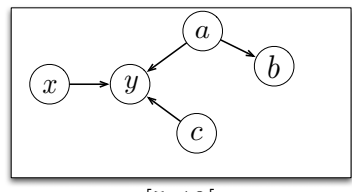

$[5,10[$

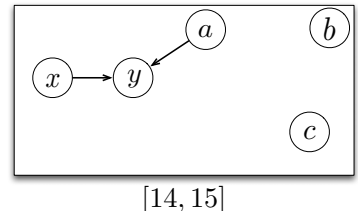

$[14,15]$ (a) Network evolution during the interval $[0,15]$

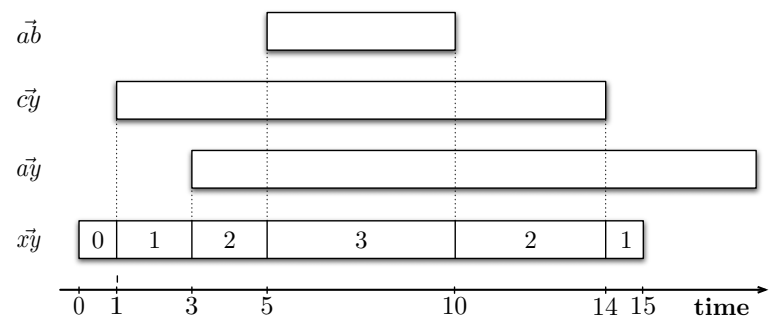

(b) Contact decomposition

Fig. 3. Dissecting a contact according to the surround indicator. In this example, the original contact $\overrightarrow{x y}$ is decomposed into six sub-intervals.

this captures well the conditions under which a contact takes place.

Note that there are other possibilities to characterize a surrounding environment of a contact. An alternative would be to simply consider the degree of a node. We do not use this representation because it does not take into account the increase in the interference probability with the increase of the number of outgoing links. Another possibility would be to consider a contact as indivisible and use the average surround indicator value computed during the entire contact duration. However, we would not be able to have an estimation of the surround stability with this solution. Yet other alternatives exist and all of them have pros and cons. As discussing the particularities of each proposal is not the goal of this paper, we leave such a comparative study for future work.

\section{THE SURROUND INDICATOR IN REAL SCENARIOS}

We use the surround indicator to characterize the spatial dimension of contacts issue from the Infocom [13] and RollerNet [16] datasets. Both datasets have been generated through contact logs between Intel iMote nodes (equipped with a Bluetooth interface). Each iMote performs regular scans and registers the MAC addresses of the responding devices around. In the Infocom dataset, iMotes were distributed to 41 students participating in the four-day Infocom student workshop in

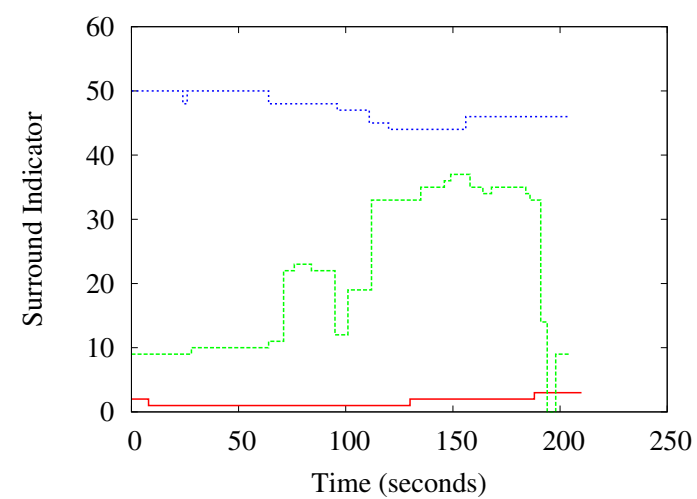

(a) Three different contact surrounds of same duration.

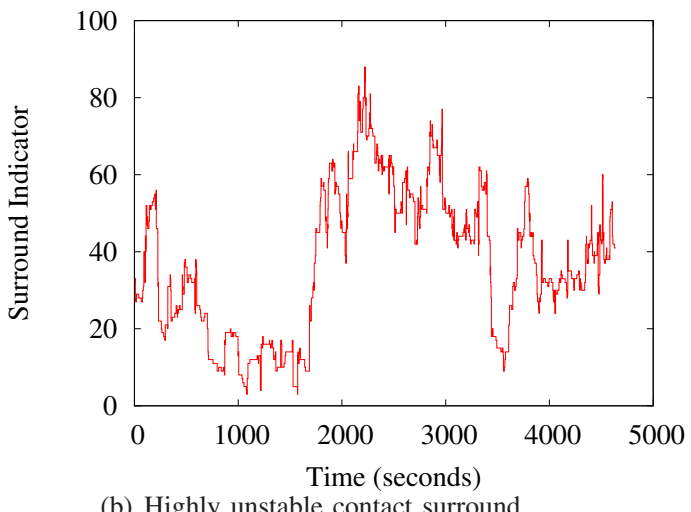

(b) Highly unstable contact surround.

Fig. 4. The surround indicator values associated to contacts arbitrarily chosen from Infocom dataset.

2005. The RollerNet dataset has been collected during a rollerblading tour in Paris. iMotes were distributed to 62 participants and the total duration of the tour was about three hours. These datasets are publicly available to the community through the Crawdad repository [19].

First, we wonder whether the two situations shown in Fig. 1 can really happen in a real setup. Fig. 4 shows the surround indicator associated to four contacts arbitrarily chosen from the Infocom dataset. We observe in Fig. 4(a) three distinct surrounding environments for contacts having the same duration. The first contact has a stable surround indicator around very low values (between 1 and 3). This situation represents a contact happening in a sparse zone. In contrast, the second contact has a stable surround indicator around large values (around 50) characterizing a much denser zone. Note that the two situations were observed in the same network. Furthermore, we could also observe in the same dataset contacts that are extremely dynamic through time, as in the third plot of Fig. 4(a). We observe this dynamics more clearly in longest contacts, as in the example shown in Fig. 4. In the remainder of this section, we will study the proportion of each situation in both the Infocom and Rollernet datasets.

We study the distribution of the surround indicator by considering the sub-intervals generated by changes in the neighborhood. Fig. 5 shows the cumulative distribution function of sub-contacts according to the surround indicator. For 


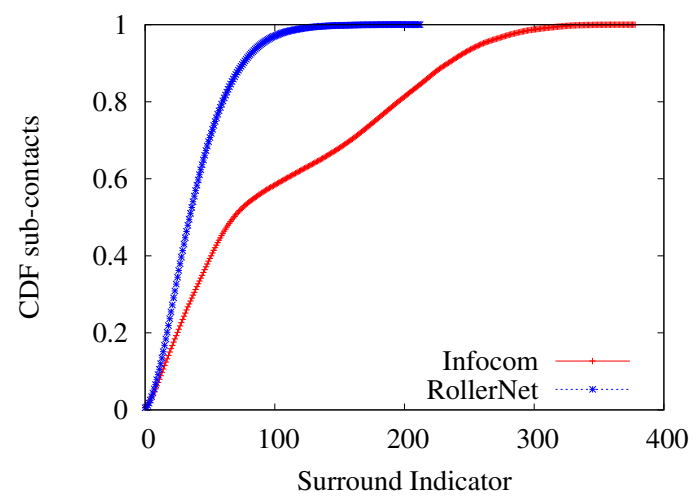

Fig. 5. Repartition of sub-contacts according to the surround indicator.

both datasets, the percentage of contacts with a null surround indicator does not exceed $0.55 \%$ for RollerNet and $0.59 \%$ for Infocom. Therefore, it is very rare that a contact happens in an isolated environment. Moreover, in extreme cases, the surround indicator reaches 212 in RollerNet and 378 in Infocom. Even if the surround indicator is just an indication of potential parallel communications, such a large number of surrounding contacts clearly shows that some zones are likely to have a limited number of working contacts, if any, due to mutual interference generated from exchanged control messages.

Let us focus now on the repartition of contact durations according to the surround indicator. Fig. 6 shows that, for both datasets, contact durations decrease when the surround indicator increases. We can explain this result by the surround evolution probability. Indeed, a contact has a small probability to observe a change of its surround in a sparse zone. As a consequence, this contact has less probability to be decomposed. On the other hand, in a dense environment, a contact has a larger probability to see one of its surrounding neighbors move during the contact time. Although it is not a surprising result, it has a major impact. By isolating a specific class of small contacts characterized by large surround indicator, we are able to compare contacts without any particular information about the medium characteristics. For example, a straightforward policy for a routing protocol would be to ignore the smallest contacts with large surround indicator when alternative paths exist.

Finally, we investigate the cumulative distribution of the contact duration according to the surround indicator, as shown in Fig. 7. Although the two curves show similar results around a surround indicator of 50 , they behave quite differently for other values. Initially, the duration of the contact with a surround indicator equal to zero represents $18 \%$ of the total contact duration in Infocom dataset when it represents only $2 \%$ in RollerNet. Consider a simple routing protocol that would ignore contacts whose surround indicator is larger than say 25 . In this case, links would be idle for more than $55 \%$ of the time in the RollerNet case while for only $35 \%$ of the time in Infocom. This result suggests that communication strategies must consider the specificities of each environment.

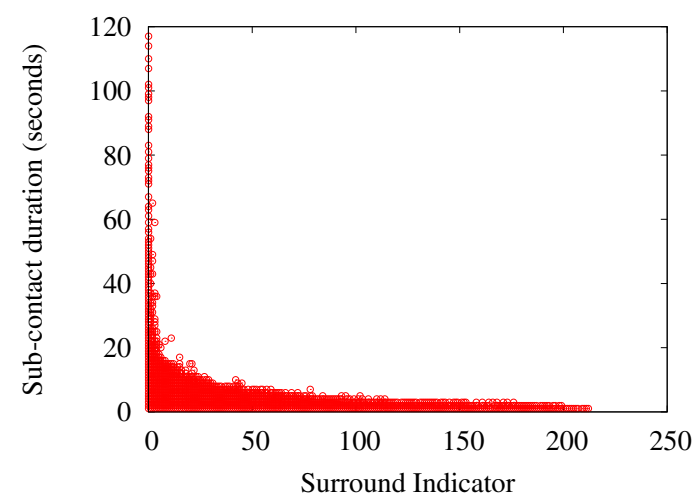

(a) RollerNet

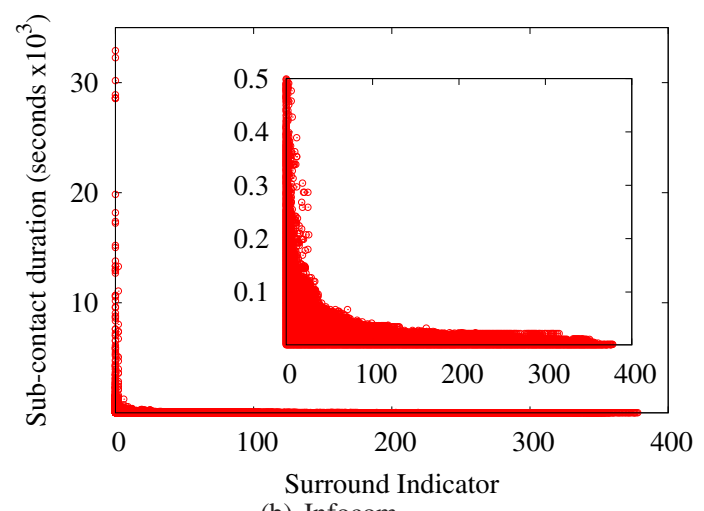

(b) Infocom

Fig. 6. Duration of contacts according to the surround indicator.

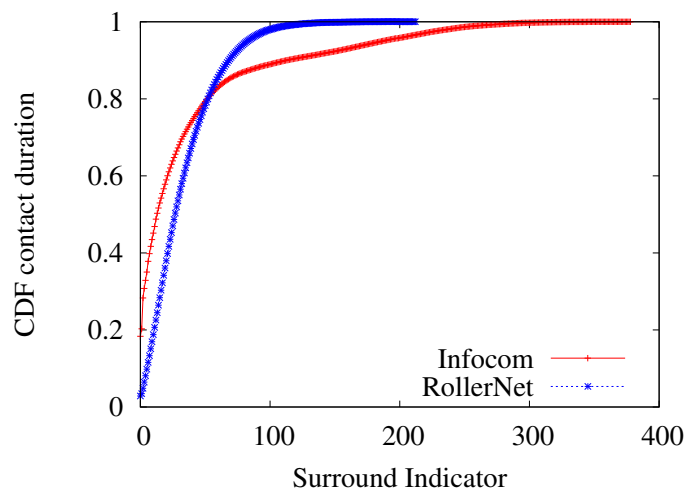

Fig. 7. Cumulative duration of contacts according to the surround indicator.

\section{DYNAMICS WITHIN CONTACTS}

We investigate now the expansion distribution to have a clue on the network dynamics. Recall that a large expansion corresponds to frequent changes of the surround indicator and reflects contact surround instability. Conversely, a small expansion corresponds to a stable environment. Fig. 8 shows the distribution of the contacts according to the expansion. In RollerNet, about $50 \%$ of the contacts have an expansion larger than 12. Similarly, in Infocom, about $50 \%$ of the contacts have an expansion larger than 16. Furthermore, although these values are relative as the datasets have different durations, the expansion reaches extreme values in both cases. In RollerNet, 


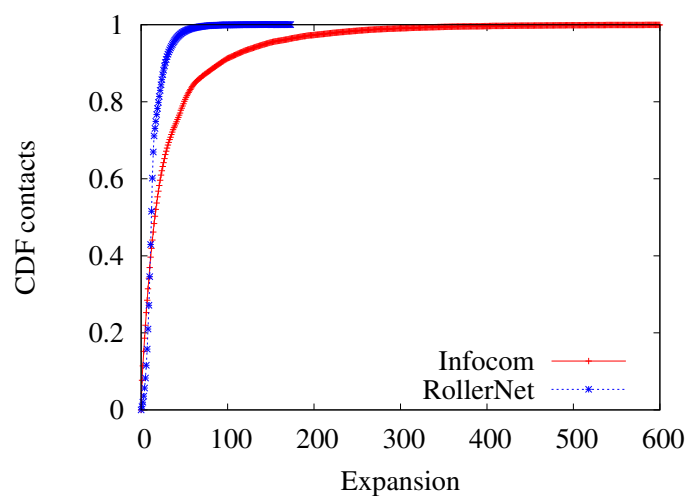

Fig. 8. Repartition of contacts according to the expansion.

the most instable contact surround has an expansion equal to 173, while it reaches 1,436 in Infocom.

It is important however to check whether the surround instability causing a high expansion reflects a minor change of the contact environment or if it is due to a significant neighborhood modification. Minor changes can be, for example, the consequence of a node that joins and leaves the contact neighborhood periodically causing a high expansion without real change in the surround. We compute, for each initial contact, the interquartile range of the surround indicator. The interquartile range is equal to the difference between the third quartile $(75 \%)$ and the first quartile $(25 \%)$ of the values taken by the surround indicator during the contact duration. It represents the dispersion of the surround indicator values. The interquartile range expresses the variability of the surround indicator associated to the same initial contact. Fig. 9 shows the distribution of the average interquartile range according to the expansion. In both datasets, when the expansion is below 60 , the average interquartile range grows with the increase of the expansion value. In other words, the instability expressed by the expansion is due to noticeable changes in the contact environment during the interval [0,60]. Note that $99 \%$ of contacts in RollerNet and $85 \%$ of contacts in Infocom have an expansion value less than 60 .

It is also interesting to observe the extreme cases. Indeed, the most unstable contact surround in RollerNet (expansion equals to 173), which has an interquartile range of 19, shows the same average interquartile range as the contacts with an expansion of 26. Also, the most unstable contact surround in Infocom (expansion equals to 1,436 ) has an interquartile range of 25 , which is lower than the average interquartile range of contacts with an expansion value of 53. In these cases, instability is due to minor but frequent changes in the contact surround.

Finally, we focus on the contact duration distribution according to the expansion values. Fig. 10 shows the durations of contacts having a particular expansion. The darker the point in the plot, the most representative the duration. In RollerNet, when we focus on the most representative values, we found that the expansion value increases with the contact duration. The accordion phenomenon that characterizes the

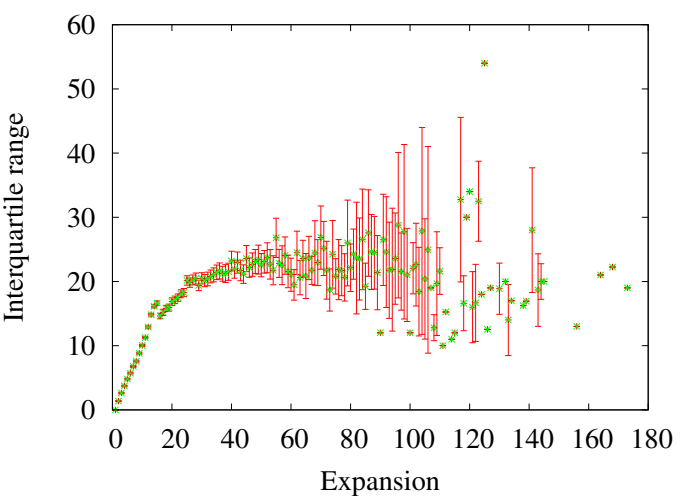

(a) RollerNet.

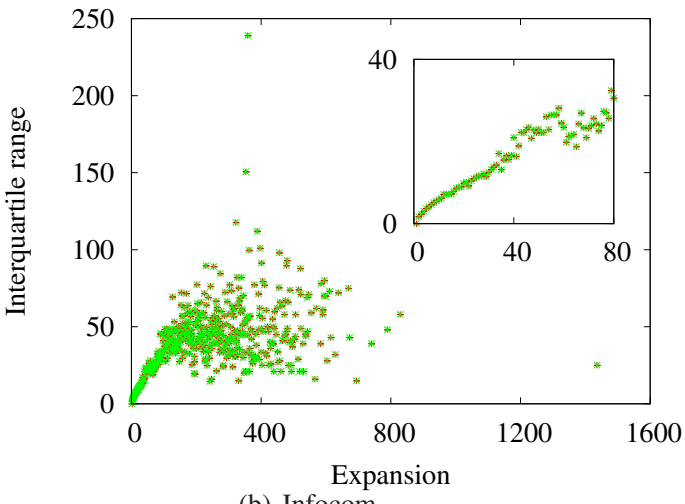

(b) Infocom.

Fig. 9. Repartition of the surround indicator interquartile range according to the expansion.

RollerNet dataset explains in part this result [16]. Indeed, such a phenomenon indicates a periodic evolution of contact surrounds. When the contact surround radically changes during the characteristic period, the longer the contact, the more varying the surround (in particular when the periodic time interval is much smaller than the contact average time). The same observation is not straightforward in the case of Infocom (cf., Fig.10(b)), where the contact duration has a different distribution showing long contacts with few surround changes. This observation can be also explained by the dataset's specificities. The Infocom experimentation records student connectivity during the four days of a conference. The students were attending to the same presentations during the day. In this context, we can understand the contact surround stability. Moreover, we note some isolated contacts (with an expansion equal to 1), which last more than 5 hours. These contacts most likely represent roommates during night. This suggests that environment properties can have a real impact on contact characterization.

\section{CONCLUSiOn AND Future WORK}

The main contribution of this paper is the introduction of the surround indicator as a metric to exhibit the spatial dimension of contacts in opportunistic networks. The surround indicator provides explicit information of the environment in which contact takes place. We use two different datasets with specific 


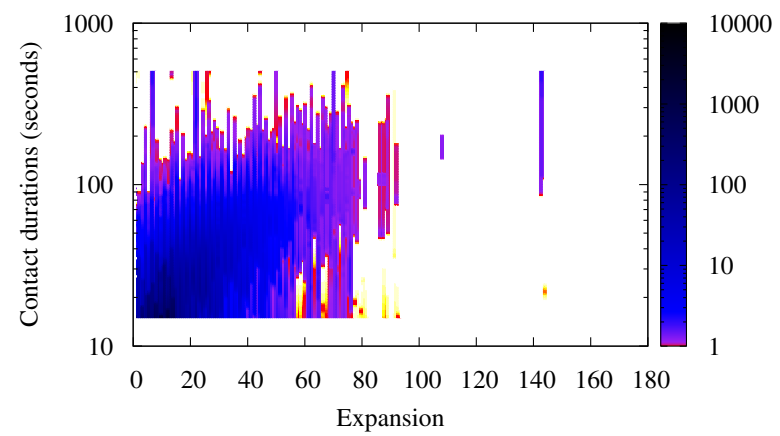

(a) RollerNet.

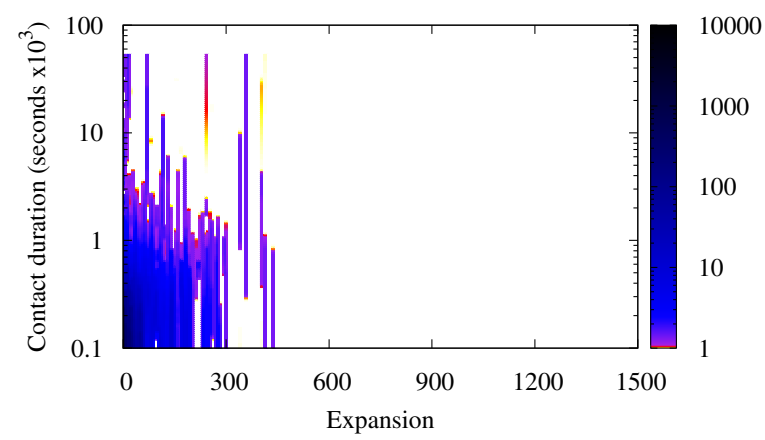

(b) Infocom.

Fig. 10. Repartition of contact durations according to the expansion.

contact environment to study the density and the expansion of the contact surround. Our analyses show that the surrounding environment of a contact is more dynamic than indicated by solely contact and intercontact times. This observation highlights the use of the proposed surround indicator as a useful yet simple metric to point out the evolution of a contact surrounding environment.

Future work includes investigating a dissemination strategy based on the surround indicator so that the next-hop selection mechanism depend on both its surrounding environment and the traffic demand in the network. The objective is to use central nodes to disseminate data when the traffic demand is low and prefer edge nodes when it is high. To this end, we intend to study the surround indicator predictability and its computation on the fly.

\section{ACKNOWLEDGMENT}

This work is partially supported by the ANR project Crowd under contract ANR-08-VERS-006 and by the European Commission in the framework of the FP7 Network of Excellence NEWCOM++ under contract 216715.

\section{REFERENCES}

[1] A. Balasubramanian, B. Levine, and A. Venkataramani, "DTN routing as a resource allocation problem," in ACM Sigcomm, Kyoto, Japan, 2007.

[2] J. Leguay, T. Friedman, and V. Conan, "Evaluating mobility pattern space routing for DTNs," in IEEE Infocom, Barcelona, Spain, 2006.

[3] A. Lindgren, A. Doria, and O. Schelén, "Probabilistic routing in intermittently connected networks," in International Workshop on Service Assurance with Partial and Intermittent Resources, Fortaleza, Brazil, 2004.

[4] T. Spyropoulos, K. Psounis, and C. S. Raghavendra, "Spray and wait: an efficient routing scheme for intermittently connected mobile networks," in ACM SIGCOMM workshop on Delay-tolerant networking, Philadelphia, Pennsylvania, USA, 2005.

[5] Q. Yuan, I. Cardei, and J. Wu, "Predict and relay: an efficient routing in disruption-tolerant networks," in ACM Mobihoc, New Orleans, LA, USA, 2009.

[6] C. Boldrini, M. Conti, and A. Passarella, "Contentplace: social-aware data dissemination in opportunistic networks," in ACM International Symposium on Modeling, Analysis and Simulation of Wireless and Mobile Systems, Vancouver, British Columbia, Canada, 2008.

[7] E. M. Daly and M. Haahr, "Social network analysis for routing in disconnected delay-tolerant manets," in ACM Mobihoc, Montreal, Quebec, Canada, 2007.
[8] P. Hui, J. Crowcroft, and E. Yoneki, "Bubble rap: social-based forwarding in delay tolerant networks," in ACM Mobihoc, Hong Kong, China, 2008.

[9] N. Sarafijanovic-Djukic, M. Piorkowski, and M. Grossglauser, "Island Hopping: Efficient Mobility-Assisted Forwarding in Partitioned Networks," in SECON, Reston, VA, 2006.

[10] S. Jung, U. Lee, A. Chang, D.-K. Cho, and M. Gerla, "Bluetorrent: Cooperative content sharing for bluetooth users," in IEEE International Conference on Pervasive Computing and Communications, White Plains, New York, USA, 2007.

[11] J. LeBrun and C.-N. Chuah, "Bluetooth content distribution stations on public transit," in International workshop on Decentralized resource sharing in mobile computing and networking, Los Angeles, California, 2006.

[12] L. McNamara, C. Mascolo, and L. Capra, "Media sharing based on colocation prediction in urban transport," in ACM Mobicom, San Francisco, California, USA, 2008.

[13] A. Chaintreau, P. Hui, C. Diot, R. Gass, and J. Scott, "Impact of human mobility on opportunistic forwarding algorithms," IEEE Transactions on Mobile Computing, vol. 6, no. 6, pp. 606-620, 2007.

[14] V. Conan, J. Leguay, and T. Friedman, "Characterizing pairwise intercontact patterns in delay tolerant networks," in International conference on Autonomic computing and communication systems, Rome, Italy, 2007.

[15] T. Karagiannis, J.-Y. L. Boudec, and M. Vojnović, "Power law and exponential decay of inter contact times between mobile devices," in ACM Mobicom. Montréal, Québec, Canada: ACM, 2007.

[16] P. U. Tournoux, J. Leguay, F. Benbadis, V. Conan, M. D. de Amorim, and J. Whitbeck, "The accordion phenomenon: Analysis, characterization, and impact on DTN routing," in IEEE Infocom, Rio de Janeiro, Brazil, 2009.

[17] A. Galati and C. Greenhalgh, "Human mobility in shopping mall environments," in ACM MobiOpp. Pisa, Italy: ACM, 2010.

[18] A. Chaintreau, A. Mtibaa, L. Massoulie, and C. Diot, "The diameter of opportunistic mobile networks," in ACM Conext. New York, NY, USA: ACM, 2007.

[19] CRAWDAD Website, http://crawdad.cs.dartmouth.edu. 